\title{
LORENTZ-INVARIANT QUANTUM FIELDS IN THE SPACE-TIME TANGENT BUNDLE
}

\author{
HOWARD E. BRANDT
}

Received 13 September 2001 and in revised form 13 March 2002

\begin{abstract}
A maximal-acceleration invariant quantum field is defined on the space-time tangent bundle with vanishing eigenvalue when acted on by the Laplace-Beltrami operator of the bundle, and the case is addressed in which the space-time is Minkowskian, and the field is Lorentz invariant. In this case, the field is shown to be automatically regularized at the Planck scale, and particle spectra are cut off at extremely high energies. The microcausality is addressed by calculating the appropriate field commutators; and it is shown that provided the adjoint field is consistently generalized, the necessary commutators are vanishing and the field is microcausal, but that there are Planck-scale modifications of the boundary of the causal domain that are significant for extremely large relative four-velocities between the separated space-time points. For vanishing relative four-velocity, the causal domain is canonical. The geometry of the causal domain indicates that near the Planck scale, causal connectivity may occur between spacelike separated points, and also at larger scales for extremely large relative four-velocities.
\end{abstract}

2000 Mathematics Subject Classification: 81T99, 53Z05, 58B20, 83E15, 22E43, 83C99, 83A05.

1. Introduction. Consider a maximal-acceleration invariant scalar field $\phi$ defined on the space-time tangent bundle and having a vanishing eigenvalue when acted on by the invariant Laplace-Beltrami operator of the bundle; namely, [12, 13, 15, 16, 17],

$$
G^{-1 / 2} \frac{\partial}{\partial x^{M}}\left(G^{1 / 2} G^{M N} \frac{\partial}{\partial x^{N}} \phi\right)=0 .
$$

Here, $G_{M N}$ is the bundle metric tensor in a coordinate basis and a point in the bundle manifold has coordinates

$$
\begin{aligned}
\left\{x^{M} ; M=0,1, \ldots, 7\right\} & =\left\{x^{\mu}, x^{m} ; \mu=0,1,2,3 ; m=4,5,6,7\right\} \\
& \equiv\left\{x^{\mu}, \rho_{0} v^{\mu} ; \mu=0,1,2,3\right\},
\end{aligned}
$$

where $x^{\mu}$ and $v^{\mu}$ are the space-time and four-velocity coordinates, respectively. (Greek indices refer to space-time, the base manifold of the bundle, and range from 0 to 3; lower case Latin indices refer to four-velocity space, the fiber manifold of the bundle, and range from 4 to 7; and upper case Latin indices refer to a point in the bundle and range from 0 to 7.) The length $\rho_{0}$ is of the 
order of the Planck length and is given by $[9,10]$

$$
\rho_{0}=\frac{c^{2}}{a_{0}}=\frac{\left(\hbar G / c^{3}\right)^{1 / 2}}{2 \pi \alpha},
$$

where $a_{0}$ is the maximum possible proper acceleration relative to the vacuum [6], $c$ is the velocity of light in vacuum, $\hbar$ is Planck's constant divided by $2 \pi$, $G$ is the universal gravitational constant, and $\alpha$ is a dimensionless constant of order unity.

The bundle structure, upon which the Laplace-Beltrami operator in (1.1) is based, follows from the invariance of maximal proper acceleration $[6,9,10]$ which restricts the differential geometric structure of the tangent bundle of space-time. For simplicity, the present work addresses a scalar field defined on the bundle and for which the invariant Laplace-Beltrami operator of the bundle acting on the field has a vanishing eigenvalue.

Equation (1.1) is invariant under the maximal acceleration group, the group of bundle-coordinate diffeomorphisms in eight dimensions [7, 8, 9, 11], and may determine possible particle excitations in the space-time tangent bundle. The ordinary inhomogeneous Lorentz group (Poincaré group) is of course a subgroup of the maximal acceleration group when the space-time is Minkowskian. A connection with canonical relativistic quantum field theory [27] can be made by considering the case in which space-time is flat and the space-time metric is Minkowskian:

$$
g_{\mu \nu}=\eta_{\mu \nu}=\left[\begin{array}{cccc}
1 & 0 & 0 & 0 \\
0 & -1 & 0 & 0 \\
0 & 0 & -1 & 0 \\
0 & 0 & 0 & -1
\end{array}\right]
$$

The bundle metric then becomes

$$
G^{M N}=\left[\begin{array}{cc}
\eta_{\mu \nu} & 0 \\
0 & \eta_{\mu \nu}
\end{array}\right]
$$

with the Minkowski metric appearing in both the base manifold and the fiber. If one substitutes (1.5) in (1.1), the scalar field equation reduces to the following eight-dimensional wave equation:

$$
\left(\square_{x}+\rho_{0}^{-2} \square_{v}\right) \phi=0,
$$

where $\square_{x}$ is the flat space-time d'Alembertian operator

$$
\square_{x}=\eta^{\mu v} \frac{\partial^{2}}{\partial x^{\mu} \partial x^{v}},
$$


and $\square_{v}$ is the analogous d'Alembertian operator defined on four-velocity space

$$
\square_{v}=\eta^{\mu v} \frac{\partial^{2}}{\partial v^{\mu} \partial v^{v}}
$$

2. Field modes. The possible separable single-mode solutions $\phi_{p}(x, v)$ to (1.6) have the form

$$
\phi_{p}(x, v)=\phi_{1}(x) \phi_{2}(v)
$$

where the dependence on the space-time coordinates $x$ is separated from the dependence on the four-velocity coordinates $v$. If one substitutes (2.1) in (1.6), then for nonvanishing $\phi_{1}(x) \phi_{2}(v)$, one obtains

$$
\frac{\square_{x} \phi_{1}(x)}{\phi_{1}(x)}+\rho_{0}^{-2} \frac{\square_{v} \phi_{2}(v)}{\phi_{2}(v)}=0 .
$$

Note that the first term of (2.2) depends only on $x$ and the second term depends only on $v$, and therefore both terms must be given by constants with the same absolute value, but with opposite signs. The constants can be defined in complete generality by $\pm(m c / \hbar)^{2}$, where $m$ is, at this point, an arbitrary constant yet to be determined. One therefore has

$$
\begin{gathered}
\frac{\square_{x} \phi_{1}(x)}{\phi_{1}(x)}=-\left(\frac{m c}{\hbar}\right)^{2}, \\
\rho_{0}^{-2} \frac{\square_{v} \phi_{2}(v)}{\phi_{2}(v)}=\left(\frac{m c}{\hbar}\right)^{2} .
\end{gathered}
$$

Possible solutions to (2.3) and (2.4) are given as

$$
\begin{aligned}
& \phi_{1}^{ \pm}(x)=\phi_{10} e^{ \pm i k x}, \\
& \phi_{2}^{ \pm}(v)=\phi_{20} e^{\mp q v},
\end{aligned}
$$

where $\phi_{10}$ and $\phi_{20}$ are constants, $k x \equiv k_{\mu} x^{\mu}, q v \equiv q_{\mu} v^{\mu}$, and $k^{\mu}, q^{\mu}$ are Lorentz four-vectors, still to be determined. For $\phi_{2}^{ \pm}(v)$ in (2.6) to be bounded, for $|q v| \rightarrow \infty$, and the exponential to be decreasing, the negative sign must be chosen in the exponent for $q v>0$ and the positive sign must be chosen for $q v<0$. Next, substituting (2.5) and (2.6) in (2.3) and (2.4), respectively, one obtains

$$
\begin{gathered}
k^{2}=\left(\frac{m c}{\hbar}\right)^{2}, \\
\rho_{0}^{-2} q^{2}=\left(\frac{m c}{\hbar}\right)^{2} .
\end{gathered}
$$


(In obtaining (2.8), we temporarily ignore the constraint on the sign of the exponent in (2.6).) Next, (2.3) can be rewritten as follows:

$$
\hbar^{2} \square_{x} \phi_{1}(x)+m^{2} c^{2} \phi_{1}(x)=0
$$

and has the same form as the ordinary massive Klein-Gordon wave equation for free scalar particles of mass $m$ [27]. The parameter $m$ can therefore be interpreted as the free-particle mass $m$. Also, for the particle four-momentum $p^{\mu}$, one has

$$
p^{2}=m^{2} c^{2}
$$

where $p^{2}=p_{\mu} p^{\mu}$. No a priori assumption is made here regarding the explicit relationship between the four-velocity coordinate $v^{\mu}$ and the particle momentum $p^{\mu}$. However, the quantum mechanical relationship between the particle fourmomentum and the associated wave frequency and wavelength is assumed to be maintained. If we compare (2.7) (which arises from (2.3) and (2.5)) with (2.10), we obtain (most simply)

$$
k=\frac{p}{\hbar} .
$$

We also assume, based on a comparison of (2.8) with (2.10), that (most simply)

$$
q=\frac{\rho_{0} p}{\hbar} .
$$

It is evident that other possibilities exist such as

$$
q^{\mu}=\left(\frac{\rho_{0}}{\hbar}\right) l_{v}^{\mu} p^{v}
$$

where $l_{v}^{\mu}$ is some linear transformation satisfying

$$
l_{\mu}^{\alpha} l_{\nu}^{\beta} \eta_{\alpha \beta}=\eta_{\mu \nu} .
$$

This and other possibilities remain to be explored. However, for simplicity, we assume (2.12) in the present work.

Next, substituting (2.11) and (2.12) in (2.5) and (2.6), respectively, and explicitly enforcing boundedness, we obtain

$$
\begin{aligned}
& \phi_{1}^{ \pm}(x)=\phi_{10} e^{ \pm i p x / \hbar}, \\
& \phi_{2}^{ \pm}(v)=\phi_{20} e^{\mp \rho_{0} p v / \hbar} \theta\left( \pm \frac{\rho_{0} p v}{\hbar}\right),
\end{aligned}
$$

where $p x=p_{\mu} x^{\mu}, p v=p_{\mu} v^{\mu}$, and $\theta(x)$ is the Heaviside step function

$$
\theta(x)= \begin{cases}1, & x>0 \\ \frac{1}{2}, & x=0 \\ 0, & x<0\end{cases}
$$


The appearance of the step function in (2.16) ensures that the correct sign choices in the exponent are implemented so that the exponential is decreasing for increasing values of the magnitude of its exponent and the field remains bounded. One must, however, demonstrate that (2.4) is still satisfied when the step function is included in (2.16). To see that (2.4) is satisfied, we substitute (2.16) in (2.4) and obtain

$$
\begin{aligned}
& \square_{v} \phi_{2}^{ \pm}(v) \\
& \quad=\phi_{20}\left(\frac{\rho_{0} m c}{\hbar}\right)^{2}\left[\delta^{\prime}\left( \pm \frac{\rho_{0} p v}{\hbar}\right)-2 \delta\left( \pm \frac{\rho_{0} p v}{\hbar}\right)+\theta\left( \pm \frac{\rho_{0} p v}{\hbar}\right)\right] e^{\mp \rho_{0} p v / \hbar},
\end{aligned}
$$

where $\delta(x)$ is the Dirac delta function and $\delta^{\prime}(x) \equiv(d / d x) \delta(x)$. We proceed to demonstrate that the two delta-function terms do not contribute because their arguments are nonvanishing and finite. First, the free-particle energy $E$ must always be positive. Also, the four-vector momentum $p^{\mu}$ in Minkowski space-time is given by

$$
p^{\mu}=\left(\frac{E}{c}, \vec{p}\right)=\left(\left[m^{2} c^{2}+|\vec{p}|^{2}\right]^{1 / 2}, \vec{p}\right),
$$

where $\vec{p}$ is the particle three-momentum. The four-velocity $v^{\mu}$, which parameterizes the fiber of the space-time tangent bundle, must lie on the four-velocity shell:

$$
v^{2}=g_{\mu \nu} v^{\mu} v^{v}=g_{\mu \nu} \frac{d x^{\mu}}{d s} \frac{d x^{v}}{d s}=\frac{g_{\mu \nu} d x^{\mu} d x^{v}}{d s^{2}}=1
$$

since the space-time interval $d s$ satisfies

$$
d s^{2}=g_{\mu \nu} d x^{\mu} d x^{\nu}
$$

Therefore, in the flat Minkowski space-time (1.4), one has

$$
v^{2}=\eta_{\mu \nu} v^{\mu} v^{v}=\left(v^{0}\right)^{2}-\left|\frac{d \vec{x}}{d s}\right|^{2}=1,
$$

and consequently, for ordinary velocity $d \vec{x} / d t$, it follows that

$$
\begin{aligned}
v^{0} & = \pm\left(1+\left(\frac{d t}{d s}\right)^{2}\left|\frac{d \vec{x}}{d t}\right|^{2}\right)^{1 / 2} \\
\left(\frac{d s}{d t}\right)^{2} & =\frac{\left(c^{2} d t^{2}-|d \vec{x}|^{2}\right)}{d t^{2}}=\left(\frac{c}{\gamma}\right)^{2}
\end{aligned}
$$

where

$$
\gamma=\left(1-\frac{|d \vec{x} / d t|^{2}}{c^{2}}\right)^{-1 / 2}
$$


Therefore, substituting (2.24) in (2.23) and using (2.25), one obtains, for the time component of four-velocity,

$$
v^{0}= \pm \gamma
$$

For classical particles and fields, four-velocity $v^{\mu}$ is future-directed; namely,

$$
v^{\mu} \equiv\left(\gamma, \gamma \frac{d \vec{x} / d t}{c}\right)
$$

corresponding to the positive sign in (2.26). However, for generality, we retain both signs in (2.26). For quantum fields, we will see that it is necessary to consider both future- and past-directed four-velocities corresponding to the positive and negative signs, respectively, in (2.26). Also, one has

$$
\vec{v}=\frac{d \vec{x}}{d s}=\frac{d t}{d s} \frac{d \vec{x}}{d t}=\frac{v^{0}}{c} \frac{d \vec{x}}{d t}
$$

or using (2.26), one obtains, for the spatial component of four-velocity,

$$
\vec{v}= \pm \gamma \frac{d \vec{x} / d t}{c}
$$

Combining (2.26) and (2.29) produces

$$
v^{\mu}= \pm\left(\gamma, \gamma \frac{d \vec{x} / d t}{c}\right)
$$

Next, using (2.19) and (2.30), one obtains

$$
p v= \pm \gamma\left(\left(m^{2} c^{2}+|\vec{p}|^{2}\right)^{1 / 2}-\frac{\vec{p} \cdot d \vec{x} / d t}{c}\right)
$$

or, equivalently,

$$
p v= \pm m c\left(1-\beta^{2}\right)^{-1 / 2} F\left(\frac{|\vec{p}|}{m c}\right) .
$$

Here, the function $F(x)$ is defined by

$$
F(x)=\left(1+x^{2}\right)^{1 / 2}-\left(\beta \cos \theta^{\prime}\right) x,
$$

where

$$
\begin{aligned}
\beta & =\frac{|d \vec{x} / d t|}{c}<1 \\
\theta^{\prime} & =\cos ^{-1}\left(\frac{\vec{p} \cdot d \vec{x} / d t}{|\vec{p}||d \vec{x} / d t|}\right) .
\end{aligned}
$$


The function $F(x)$ of (2.33) has a minimum at $x=x_{\min }$ for

$$
0=\frac{\partial F}{\partial x}=x\left(1+x^{2}\right)^{-1 / 2}-\beta \cos \theta^{\prime}
$$

or

$$
x_{\min }=\beta \cos \theta^{\prime}\left(1-\beta^{2} \cos ^{2} \theta^{\prime}\right)^{-1 / 2} .
$$

Substituting (2.37) in (2.33), one has

$$
F\left(x_{\min }\right)=\left(1-\beta^{2} \cos ^{2} \theta^{\prime}\right)^{1 / 2} .
$$

Therefore, if one substitutes (2.38) in (2.32) and uses (2.34), we obtain the following inequalities:

$$
\begin{aligned}
& p v>+m c\left[\frac{\left(1-\beta^{2} \cos ^{2} \theta^{\prime}\right)}{\left(1-\beta^{2}\right)}\right]^{1 / 2}>+m c, \\
& p v<-m c\left[\frac{\left(1-\beta^{2} \cos ^{2} \theta^{\prime}\right)}{\left(1-\beta^{2}\right)}\right]^{1 / 2}<-m c .
\end{aligned}
$$

Therefore, for nonvanishing $m$, it is true that

$$
\delta\left( \pm \frac{\rho_{0} p v}{\hbar}\right)=0, \quad \delta^{\prime}\left( \pm \frac{\rho_{0} p v}{\hbar}\right)=0
$$

Substituting (2.40) in (2.18) and using (2.16), one obtains

$$
\square_{v} \phi_{2}^{ \pm}(v)=\left(\frac{\rho_{0} m c}{\hbar}\right)^{2} \phi_{2}^{ \pm}(v)
$$

Comparing (2.41) with (2.4), we can conclude that $\phi_{2}^{ \pm}(v)$, given by (2.16), does in fact satisfy (2.4).

Next, substituting (2.15) and (2.16) in (2.1), we obtain the following positive and negative frequency mode solutions to (1.6):

$$
\phi_{p}^{ \pm}(x, v)=\phi_{0} e^{\mp i p x / \hbar} e^{\mp \rho_{0} p v / \hbar} \theta\left( \pm \frac{\rho_{0} p v}{\hbar}\right)
$$

where $\phi_{0}$ is a constant and $p^{0}$ is positive and given by

$$
p^{0}=\left(m^{2} c^{2}+|\vec{p}|^{2}\right)^{1 / 2} \text {. }
$$

3. Scalar quantum field. One obtains the corresponding free relativistic scalar quantum field $\phi(x, v)$ by integrating over the ordinary three-momentum of the invariant positive and negative frequency modes $\phi_{p}^{ \pm}(x, v)$ of (2.42), 
including appropriate creation and annihilation operators [27]. It follows from (2.26), (2.30), (2.31), (2.39), (2.42), and the property of the Heaviside step function of (2.17) that, for particles (positive frequency) and antiparticles (negative frequency), nonvanishing support is provided for $v^{0}=+\gamma$ and for $v^{0}=-\gamma$, respectively. Thus, $p v$ is effectively positive for a particle and negative for an antiparticle. Hence, the free relativistic scalar quantum field $\phi(x, v)$ in the space-time tangent bundle is given by $[12,13,15,17]$

$$
\begin{aligned}
\phi(x, v)=2 \int \frac{d^{3} p}{(2 \pi)^{3 / 2}\left(2 p^{0}\right)^{1 / 2}}[ & e^{-i p x / \hbar} e^{-\rho_{0} p v / \hbar} \theta\left(\frac{\rho_{0} p v}{\hbar}\right) a(p) \\
& \left.+e^{i p x / \hbar} e^{\rho_{0} p v / \hbar} \theta\left(-\frac{\rho_{0} p v}{\hbar}\right) a^{\dagger}(p)\right],
\end{aligned}
$$

in which $a(p)$ and $a^{\dagger}(p)$ are the annihilation and creation operators, respectively, for particles and their uncharged antiparticles, and the operators satisfy the usual commutation relations [27]

$$
\begin{gathered}
{\left[a(p), a^{\dagger}\left(p^{\prime}\right)\right]=\delta^{3}\left(p-p^{\prime}\right),} \\
{\left[a(p), a\left(p^{\prime}\right)\right]=0,} \\
{\left[a^{\dagger}(p), a^{\dagger}\left(p^{\prime}\right)\right]=0 .}
\end{gathered}
$$

To accommodate normalization associated with the four-velocity coordinate of the space-time tangent bundle, the integration measure in the tangent space must be appropriately chosen. This would be important, for example, in calculating the particle number density, or the average particle four-velocity. Also, it is important to emphasize that throughout the present work, the field describes a neutral particle which is its own antiparticle. In (3.1), we choose the field normalization to agree with that a canonical free relativistic scalar quantum field [27] in the limit of infinite maximal proper acceleration $a_{0}$ or, equivalently, vanishing $\rho_{0}$. Thus, using (2.17) and (3.1), we can see that

$$
\phi(x, v) \underset{\rho_{0} \rightarrow 0}{\longrightarrow} \int \frac{d^{3} p}{(2 \pi)^{3 / 2}\left(2 p^{0}\right)^{1 / 2}}\left[e^{-i p x / \hbar} a(p)+e^{i p x / \hbar} a^{\dagger}(p)\right]
$$

which is the appropriate limit for canonical relativistic quantum field theory.

In the regions of nonvanishing support, both terms of (3.1) effectively have the decreasing exponential factor $\exp \left(-\rho_{0}|p v| / \hbar\right)$, which can be rewritten using (1.3) and (2.31) as

$$
\begin{aligned}
& \exp \left(-\rho_{0} \frac{|p v|}{\hbar}\right) \\
& \quad=\exp \left(-\frac{1}{2 \pi \alpha} \frac{\gamma m}{m_{\mathrm{pl}}}\left\{\left[1+\left(\frac{|\vec{p}|}{m c}\right)^{2}\right]^{1 / 2}-\frac{\vec{p} \cdot \overrightarrow{d x} / d t}{m c^{2}}\right\}\right),
\end{aligned}
$$


where the Planck mass $m_{\mathrm{pl}}$ is given by

$$
m_{\mathrm{pl}}=\left(\frac{\hbar c}{G}\right)^{1 / 2}
$$

The spectrum is exponentially cut off beyond the Planck mass, resulting in an automatic field regularization.

4. Microcausality. Next, we address the subject of microcausality [2, 3, 4, $5,18,22,23,24,25,27]$ for Lorentz-invariant quantum fields in the space-time tangent bundle with a Minkowski space-time base manifold, by calculating the appropriate field commutators $[14,16]$. We proceed to argue that provided the adjoint operation on the fields is consistently generalized to include fourvelocity sign-inversion, the necessary commutators are vanishing and the field is microcausal. There are, however, Planck-scale modifications of the causal domain that only become significant for extremely large relative four-velocities at the separated space-time points. For vanishing relative four-velocities, the causal domain is canonical. The generalized adjoint operation is to be consistent with the facts that (1) the time component of four-velocity is positive for particles and negative for antiparticles, (2) the adjoint operation on the scalar field corresponds to charge conjugation, which replaces particles by antiparticles and antiparticles by particles, and (3) the neutral scalar particle is its own antiparticle.

Using (3.1), it can be shown that outside the causal domain, one has [16]

$$
\begin{aligned}
& {\left[\phi(x, v), \phi\left(x^{\prime}, v^{\prime}\right)\right]} \\
& =\frac{m c \hbar}{\pi^{2}}\left[\theta\left(\frac{\rho_{0} m c v^{0}}{\hbar}\right) \theta\left(-\frac{\rho_{0} m c v^{0^{\prime}}}{\hbar}\right)-\theta\left(\frac{\rho_{0} m c v^{0^{\prime}}}{\hbar}\right) \theta\left(-\frac{\rho_{0} m c v^{0}}{\hbar}\right)\right] \\
& \quad \times \frac{K_{1}\left((m c / \hbar)\left[-\left(x-x^{\prime}-i \rho_{0}\left(v-v^{\prime}\right)\right)^{2}\right]^{1 / 2}\right)}{\left[-\left(x-x^{\prime}-i \rho_{0}\left(v-v^{\prime}\right)\right)^{2}\right]^{1 / 2}},
\end{aligned}
$$

where $K_{1}$ is the modified Bessel function of the third kind and of order one [19]. Alternatively, using (3.1), one also obtains [16]

$$
\begin{aligned}
& {\left[\phi(x, v), \phi\left(x^{\prime}, v^{\prime}\right)\right]} \\
& =4\left[\theta\left(\frac{\rho_{0} m c v^{0}}{\hbar}\right) \theta\left(-\frac{\rho_{0} m c v^{0^{\prime}}}{\hbar}\right) \int \frac{d^{3} p}{(2 \pi)^{3}\left(2 p^{0}\right)} e^{-i p\left(x-x^{\prime}\right) / \hbar} e^{-\rho_{0} p\left(v-v^{\prime}\right) / \hbar}\right. \\
& \left.\quad-\theta\left(\frac{\rho_{0} m c v^{0^{\prime}}}{\hbar}\right) \theta\left(-\frac{\rho_{0} m c v^{0}}{\hbar}\right) \int \frac{d^{3} p}{(2 \pi)^{3}\left(2 p^{0}\right)} e^{i p\left(x-x^{\prime}\right) / \hbar} e^{-\rho_{0} p\left(v^{\prime}-v\right) / \hbar}\right],
\end{aligned}
$$


in which the integrals remain unevaluated. Equation (4.2) can be used for those cases in which the evaluation of the commutator depends only on the properties of the Heaviside step function of (2.17), and in which the arguments leading to (4.1) may not apply.

Here, except for possible Planck-scale effects, spacelike space-time separations are of primary interest since microcausality is the issue. It is well to note explicitly that the modified Bessel function and also the reciprocal of the denominator in (4.1) both become singular when the Bessel-function argument is vanishing, namely, when $[14,16]$

$$
\left[x-x^{\prime}-i \rho_{0}\left(v-v^{\prime}\right)\right]^{2}=0 .
$$

Equivalently, one has

$$
\begin{gathered}
\left(x-x^{\prime}\right)^{2}=\rho_{0}^{2}\left(v-v^{\prime}\right)^{2}, \\
\rho_{0}\left(v-v^{\prime}\right) \cdot\left(x-x^{\prime}\right)=0 .
\end{gathered}
$$

Thus, if the relative four-velocity is vanishing, the modified Bessel function is singular on the light cone $\left(x-x^{\prime}\right)^{2}=0$ as in the canonical theory. Equations (4.3), (4.4), and (4.5) exhibit explicitly the Planck-scale effects on the boundary of the causal domain. Note that equal times are a special case of spacelike separations $\left(x-x^{\prime}\right)^{2}<0$ and, in accordance with special relativity, it is clearly true that at equal times, the ordinary velocities at the separated space-time points must be the same in order to maintain simultaneity, and this is consistent with the corresponding four-velocities being equal in (4.4). Just as the light cone $\left(x-x^{\prime}\right)^{2}=0$ separates the causal timelike domain from the acausal spacelike domain for a canonical relativistic field in Minkowski space-time, the six-dimensional hypersurface defined by (4.4) and (4.5) also separates the causal domain from the acausal domain for the field $\phi(x, v)$ in the Minkowskispace-time tangent bundle.

Equations (4.4) and (4.5) can also be written as

$$
\begin{gathered}
\left(\Delta x^{0}\right)^{2}=|\Delta \vec{x}|^{2}+\rho_{0}^{2}\left(\Delta v^{0}\right)^{2}-\rho_{0}^{2}|\Delta \vec{v}|^{2}, \\
\rho_{0} \Delta v^{0} \Delta x^{0}=\rho_{0} \Delta \vec{v} \cdot \Delta \vec{x},
\end{gathered}
$$

respectively, where the components of space-time separation are $\Delta x^{0}=x^{0}-$ $x^{0^{\prime}}$ and $\Delta \vec{x}=\vec{x}-\vec{x}^{\prime}$ and the components of relative four-velocity are $\Delta v^{0}=$ $v^{0}-v^{0^{\prime}}$ and $\Delta \vec{v}=\vec{v}-\vec{v}^{\prime}$. Next, multiplying (4.6) by $\left(\Delta x^{0}\right)^{2}$ and substituting (4.7) into the result, one obtains

$$
\left(\Delta x^{0}\right)^{4}-\left(|\Delta \vec{x}|^{2}-\rho_{0}^{2}|\Delta \vec{v}|^{2}\right)\left(\Delta x^{0}\right)^{2}-\rho_{0}^{2}|\Delta \vec{v} \cdot \Delta \vec{x}|^{2}=0 .
$$


According to the quadratic formula, it then follows from (4.8) that

$$
\Delta x^{0}= \pm\left(|\Delta \vec{x}|^{2}-\rho_{0}^{2}|\Delta \vec{v}|^{2}\right)^{1 / 2}\left[\frac{1}{2} \pm \frac{1}{2}\left(1+\left(\frac{2 \rho_{0} \Delta \vec{v} \cdot \Delta \vec{x}}{|\Delta \vec{x}|^{2}-\rho_{0}^{2}|\Delta \vec{v}|^{2}}\right)^{2}\right)^{1 / 2}\right]^{1 / 2}
$$

Choosing the positive sign inside the bracket and preceding the radical, so that $\Delta x^{0}$ is real, (4.9) can also be written as follows:

$$
T= \pm\left\{\frac{1}{2}\left(X^{2}-1\right)+\frac{1}{2}\left[X^{4}+2\left(2 \cos ^{2} \theta-1\right) X^{2}+1\right]^{1 / 2}\right\}^{1 / 2},
$$

where

$$
\begin{gathered}
T \equiv \frac{\Delta x^{0}}{\rho_{0}|\Delta \vec{v}|}, \\
X \equiv \frac{|\Delta \vec{x}|}{\rho_{0}|\Delta \vec{v}|}, \\
\theta \equiv \cos ^{-1}\left(\frac{\Delta \vec{v} \cdot \Delta \vec{x}}{|\Delta \vec{v}||\Delta \vec{x}|}\right) .
\end{gathered}
$$

Equation (4.13) defines the angle between the spatial component of relative four-velocity and the spatial-separation vector. Also, (4.7) can be written as

$$
V=\frac{X}{T} \cos \theta
$$

where

$$
V \equiv \frac{\rho_{0} \Delta v^{0}}{\rho_{0}|\Delta \vec{v}|}
$$

Substituting (4.10) in (4.14), one obtains

$$
V= \pm X \cos \theta\left\{\frac{1}{2}\left(X^{2}-1\right)+\frac{1}{2}\left[X^{4}+2\left(2 \cos ^{2} \theta-1\right) X^{2}+1\right]^{1 / 2}\right\}^{-1 / 2}
$$

Also, using (4.10) and (4.14), one has

$$
V= \pm\left(\frac{1+T^{2}}{1+T^{2} \sec ^{2} \theta}\right)^{1 / 2}
$$

Equations (4.10), (4.16), and (4.17) are plotted in Figures 4.1, 4.2, and 4.3, respectively, for several values of $\theta$. These figures correspond to representative slices of the six-dimensional hypersurface which is the boundary (4.4) and (4.5) of the causal domain [14].

Observe in Figure 4.1 that for a large spatial component of relative fourvelocity $|\Delta \vec{v}|$ (see (4.10), (4.11), and (4.12)), the Planck-scale effects [16] become manifest and the causal domain penetrates significantly into the canonical spacelike region for spatial separations within several multiples of $\rho_{0}|\Delta \vec{v}|$. The 


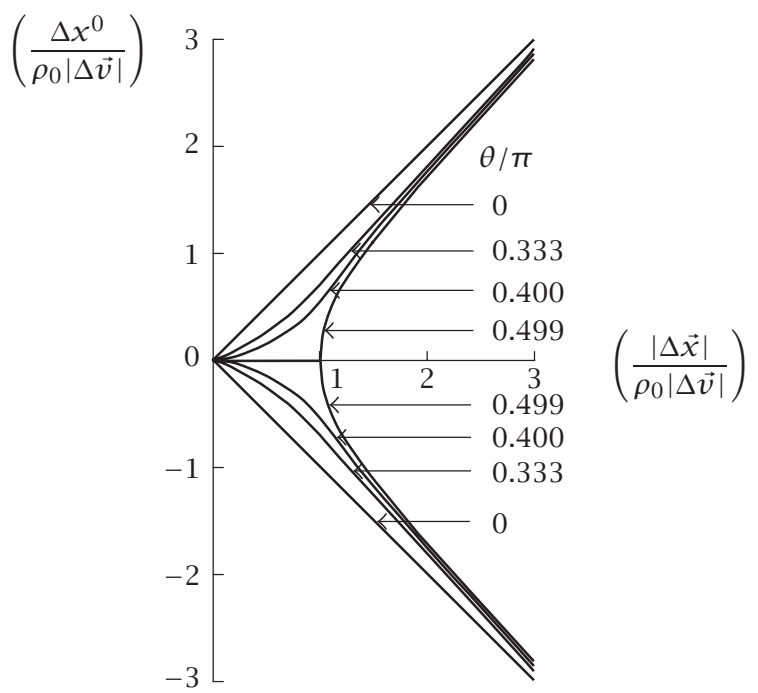

Figure 4.1. Causal boundary equation (4.10): $\left(\Delta x^{0} / \rho_{0}|\Delta \vec{v}|\right)$ as a function of $\left(|\Delta \vec{x}| / \rho_{0}|\Delta \vec{v}|\right)$ for various values of the angle $\theta$ between the spatial component $\Delta \vec{v}$ of relative four-velocity and the spatialseparation vector $\Delta \vec{x}$.

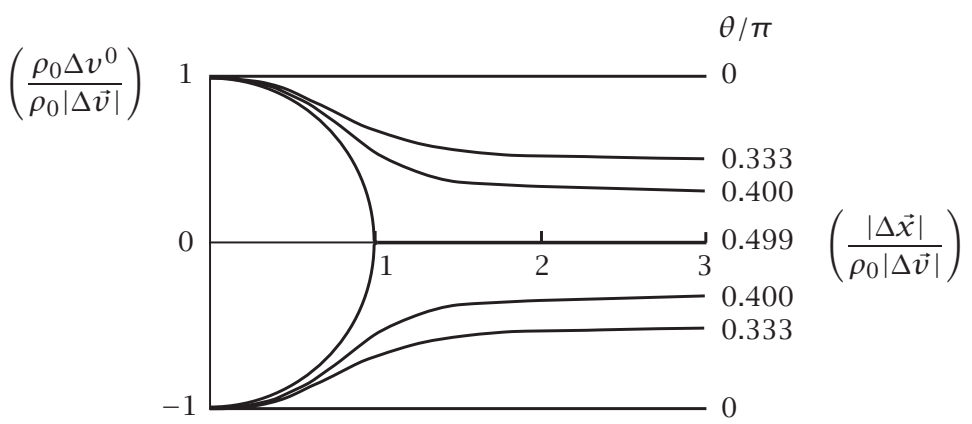

FIGURE 4.2. Causal boundary equation (4.16): $\left(\rho_{0} \Delta v^{0} / \rho_{0}|\Delta \vec{v}|\right)$ as a function of $\left(|\Delta \vec{x}| / \rho_{0}|\Delta \vec{v}|\right)$ for various values of the angle $\theta$ between the spatial component $\Delta \vec{v}$ of relative four-velocity and the spatialseparation vector $\Delta \vec{x}$.

effect is least when the spatial part of the relative four-velocity $\Delta \vec{v}$ is nearly parallel or antiparallel to the spatial-separation vector $\Delta \vec{x}$ corresponding to $\theta \approx 0$ or $\pi$. The effect is largest when $\theta=\pi / 2$ and the spatial part of the relative four-velocity is perpendicular to the spatial-separation vector. For $\theta=\pi / 2$ in (4.10), $T$ is complex for $X<1$ and the causal boundary is on the hyperbola $T= \pm\left(X^{2}-1\right)^{1 / 2}$, which corresponds to the single-sheeted four-hyperboloid 


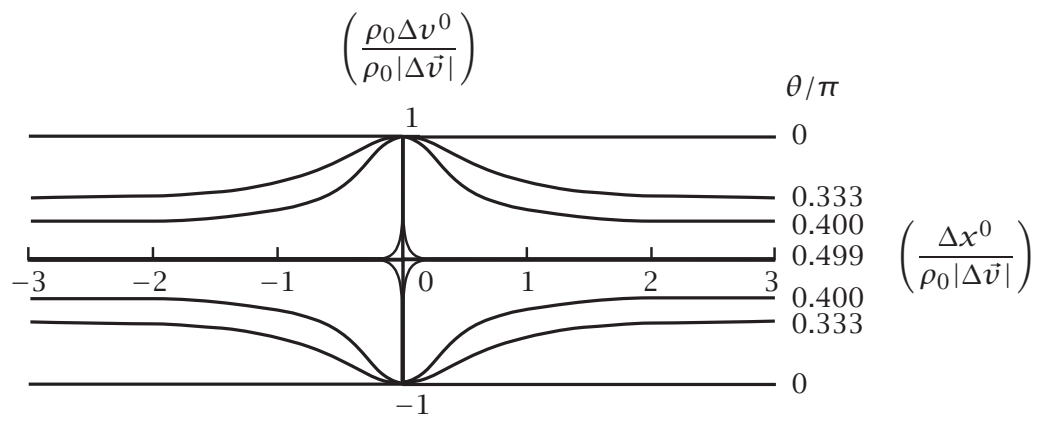

FiguRE 4.3. Causal boundary equation (4.17): $\left(\rho_{0} \Delta v^{0} / \rho_{0}|\Delta \vec{v}|\right)$ as a function of $\left(\Delta x^{0} / \rho_{0}|\Delta \vec{v}|\right)$ for various values of the angle $\theta$ between the spatial component $\Delta \vec{v}$ of relative four-velocity and the spatialseparation vector $\Delta \vec{x}$.

[28] in space-time:

$$
\left(\Delta x^{1}\right)^{2}+\left(\Delta x^{2}\right)^{2}+\left(\Delta x^{3}\right)^{2}-\left(\Delta x^{0}\right)^{2}=\rho_{0}^{2}|\Delta \vec{v}|^{2} .
$$

From (4.10), it follows that

$$
\frac{d T}{d X}_{\left.\right|_{X=0}}= \pm \cos \theta
$$

Thus, in Figure 4.1, the pointed section of the hypersurface near the singular point at the origin becomes sharper as $\theta$ ranges from 0 to $\pi / 2$. In the limit as $\theta \rightarrow \pi / 2$, it becomes an infinitesimally thin cusp tangent (above and below) to the segment $0 \leq X \leq 1$ of the $X$-axis at $T=0$. The rest of the causal boundary for $\theta \rightarrow \pi / 2$ becomes tangent to the hyperbola $T= \pm\left(X^{2}-1\right)^{1 / 2}$. The segment corresponds to the simultaneous $\left(\Delta x^{0}=0\right)$ and spacelike threesphere $\left(\Delta x^{1}\right)^{2}+\left(\Delta x^{2}\right)^{2}+\left(\Delta x^{3}\right)^{2}=\rho_{0}^{2}|\Delta \vec{v}|^{2}$ and its interior, and the hyperbola corresponds to the four-hyperboloid equation (4.18) (see [28]). All points on the tangential infinitesimally thin cusp (as well as those on the tangent to the hyperbola) lie on the hypersurface on which the field commutator of (4.1) is singular [16], and thus correspond to causal space-time separations (even though the points of the three-sphere and its interior are simultaneous). The geometry indicates that at the Planck scale, causal connectivity of the fields may occur between spacelike separated points and also at larger scales for extremely large relative four-velocities. This suggests the possibility that for nominal values of the spatial component of relative four-velocity, causally connected Planck size spatially extended excitations (such as strings or brans) may not be 
incompatible with the structure of the causal domain near the Planck scale. The spatial extent of the distortions of the canonical causal domain scales linearly with the magnitude of the spatial component of relative four-velocity and may extend well beyond the Planck scale for extremely large relative four-velocities. However, extremely large relative four-velocities also require extremely large four-velocities for which the field is exponentially cut off as in (3.4).

Proceeding with the evaluation of (4.1) in the limit of infinite maximal proper acceleration, using (1.3) and (2.17), one gets

$$
\left[\phi(x, v), \phi\left(x^{\prime}, v^{\prime}\right)\right] \underset{\rho_{0} \rightarrow 0}{\longrightarrow} 0
$$

as must be the case for consistency with the canonical scalar field [27], which satisfies microcausality. Because of the Heaviside step functions in (4.2), one can immediately see, using (2.17) and (2.30), that

$$
\left[\phi(x, v), \phi\left(x^{\prime}, v^{\prime}\right)\right]_{\mid v^{0} v^{0^{\prime}>0}}=0 .
$$

Equation (4.21) is consistent with microcausality. That (4.21) also applies when the fields have four-velocities that are both past-directed need not be a priori inconsistent with relativistic causality.

If $v$ is future-directed $\left(v^{0}>0\right)$ and $v^{\prime}$ is past-directed $\left(v^{0^{\prime}}<0\right)$, one obtains, using (2.17) and (2.30) in (4.1), the nonvanishing value

$$
\begin{aligned}
& {\left[\phi(x, v), \phi\left(x^{\prime}, v^{\prime}\right)\right]_{\mid v^{0}>0, v^{0^{\prime}}<0}} \\
& \quad=\frac{m c \hbar}{\pi^{2}} \frac{K_{1}\left((m c / \hbar)\left[-\left(x-x^{\prime}-i \rho_{0}\left(v-v^{\prime}\right)\right)^{2}\right]^{1 / 2}\right)}{\left[-\left(x-x^{\prime}-i \rho_{0}\left(v-v^{\prime}\right)\right)^{2}\right]^{1 / 2}} .
\end{aligned}
$$

Equation (4.22) is apparently not inconsistent with microcausality because the four-velocities at the separated space-time points are oppositely directed in time, in which case the concept of causality loses any obvious meaning. Also, the associated four-velocity shells are disjoint. The space-time points are however causally separated on the singular hypersurface on which the commutator becomes singular [16]. Also, note that because the modified Bessel function $K_{1}$ decreases exponentially for large arguments [1], the right-hand side of (4.22) will typically (except for extremely large relative four-velocities) be very small for spacelike separations exceeding several multiples of the Compton wavelength $\hbar / m c$. Because $\rho_{0}$ is only of the order of the Planck length, the effect of the imaginary parts in the denominator and in the argument of the modified Bessel function can be expected to be negligible, except at extremely large separations in the four-velocity tangent space of the space-time tangent bundle for 
which Planck-scale structure becomes manifest. Analogously to the derivation of (4.22), one obtains

$$
\begin{aligned}
& {\left[\phi(x, v), \phi\left(x^{\prime}, v^{\prime}\right)\right]_{\mid v^{0}<0, v^{0^{\prime}}>0}} \\
& \quad=-\frac{m c \hbar}{\pi^{2}} \frac{K_{1}\left((m c / \hbar)\left[-\left(x-x^{\prime}-i \rho_{0}\left(v-v^{\prime}\right)\right)^{2}\right]^{1 / 2}\right)}{\left[-\left(x-x^{\prime}-i \rho_{0}\left(v-v^{\prime}\right)\right)^{2}\right]^{1 / 2}},
\end{aligned}
$$

which is also apparently not inconsistent with microcausality for the same reasons already given for (4.22).

Next, note that without any qualifications on the canonical adjoint operator, the field $\phi$ of (3.1) is apparently not Hermitian. It is important for the consistent interpretation of the commutators involving the ordinary adjoint field $\phi^{\dagger}(x, v)[16]$ to introduce a generalized adjoint field $(\phi(x, v))^{\dagger}$ as the charge conjugate field obtained by particle-antiparticle conjugation analogously to the canonical case [27], with particles replaced by antiparticles and antiparticles replaced by particles, and also to recall that particles and antiparticles only have nonvanishing support for $v^{0}>0$ and $v^{0}<0$, respectively, in (3.1). Although charge conjugation has nothing to do with the space or time coordinates (as in the canonical theory), it apparently does have to do with the tangent space coordinates of the space-time tangent bundle because particles have nonvanishing support for future-directed four-velocities, while antiparticles have nonvanishing support for past-directed four-velocities [12, 13, 15, 17]. It is thus logical to define the charge-conjugate field $\phi_{c}(x, v)$ and the generalized adjoint $(\phi(x, v))^{\dagger}$ of the quantum field $\phi(x, v)$ by [16]

$$
\phi_{c}(x, v)=(\phi(x, v))^{\dagger} \equiv \phi^{\dagger}(x,-v) .
$$

Equation (4.24) is compatible with the canonical adjoint operation [27] since, in the latter, the fields are independent of four-velocity, and therefore fourvelocity does not even enter into consideration. Next, substituting (3.1) in the second equality of (4.24), it follows that Hermiticity is upheld in a generalized sense; namely,

$$
(\phi(x, v))^{\dagger} \equiv \phi(x, v) .
$$

Equations (4.24) and (4.25), analogously to the canonical case [27], guarantee the identity of the particle and antiparticle states for the neutral scalar quantum field; namely,

$$
\phi_{c}(x, v)=\phi(x, v) .
$$

Equation (4.26) is a consistent generalization of the corresponding canonical relation [27]. In the latter, of course, four-velocity does not enter. One is however reminded of the Stückelberg-Feynman idea that an antiparticle can be interpreted as a particle going backward in time [20, 21, 26]. 
Note also that by using (3.1), (4.24), and (4.25), one can show that [16]

$$
\begin{aligned}
& {\left[\phi(x, v),\left(\phi\left(x^{\prime}, v^{\prime}\right)\right)^{\dagger}\right]} \\
& =\frac{m c \hbar}{\pi^{2}}\left[\theta\left(\frac{\rho_{0} m c v^{0}}{\hbar}\right) \theta\left(-\frac{\rho_{0} m c v^{0^{\prime}}}{\hbar}\right)-\theta\left(-\frac{\rho_{0} m c v^{0}}{\hbar}\right) \theta\left(\frac{\rho_{0} m c v^{0^{\prime}}}{\hbar}\right)\right] \\
& \quad \times \frac{K_{1}\left((m c / \hbar)\left[-\left(x-x^{\prime}-i \rho_{0}\left(v-v^{\prime}\right)\right)^{2}\right]^{1 / 2}\right)}{\left[-\left(x-x^{\prime}-i \rho_{0}\left(v-v^{\prime}\right)\right)^{2}\right]^{1 / 2}} .
\end{aligned}
$$

With the generalized adjoint operation, the appropriate field commutators are all vanishing and are consistent with microcausality [16]. The Planck-scale structure does manifest itself in modifications of the boundary of the causal domain, but significant modifications appear only for extremely large relative four-velocities at the separated space-time points.

Since the fields themselves may not correspond to physical observables, it is important to consider bilinear forms of the fields, corresponding to local observables [22]. Microcausality requires that observables commute for spacelike separations in space-time. A possible operator corresponding to a local observable is defined by

$$
O(x, v) \equiv(\phi(x, v))^{\dagger} \phi(x, v),
$$

or substituting (4.24) in (4.28), one has for the observable the following expression:

$$
O(x, v)=\phi^{\dagger}(x,-v) \phi(x, v) .
$$

Using (4.29), (4.24), and (4.28), one obtains

$$
O^{\dagger}(x, v) \equiv(\phi(x, v))^{\dagger}\left(\phi^{\dagger}(x,-v)\right)^{\dagger}=\phi^{\dagger}(x,-v) \phi(x, v)=O(x, v) .
$$

Thus, the observable is Hermitian in the generalized sense.

Next, consider the commutator $\left[O(x, v), O\left(x^{\prime}, v^{\prime}\right)\right]$ which must be vanishing for spacelike separations in space-time and future-directed four-velocities if consistency with microcausality is to be upheld. First, using (4.28), one has

$$
\left[O(x, v), O\left(x^{\prime}, v^{\prime}\right)\right]=\left[(\phi(x, v))^{\dagger} \phi(x, v),\left(\phi\left(x^{\prime}, v^{\prime}\right)\right)^{\dagger} \phi\left(x^{\prime}, v^{\prime}\right)\right]
$$

Then, substituting (4.24) in (4.31), one obtains

$$
\left[O(x, v), O\left(x^{\prime}, v^{\prime}\right)\right]=\left[\phi^{\dagger}(x,-v) \phi(x, v), \phi^{\dagger}\left(x^{\prime},-v^{\prime}\right) \phi\left(x^{\prime}, v^{\prime}\right)\right]
$$


If one expands (4.32), it becomes

$$
\begin{aligned}
{\left[O(x, v), O\left(x^{\prime}, v^{\prime}\right)\right]=} & \phi^{\dagger}(x,-v) \phi^{\dagger}\left(x^{\prime},-v^{\prime}\right)\left[\phi(x, v), \phi\left(x^{\prime}, v^{\prime}\right)\right] \\
& +\phi^{\dagger}(x,-v)\left[\phi(x, v), \phi^{\dagger}\left(x^{\prime},-v^{\prime}\right)\right] \phi\left(x^{\prime}, v^{\prime}\right) \\
& +\phi^{\dagger}\left(x^{\prime},-v^{\prime}\right)\left[\phi^{\dagger}(x,-v), \phi\left(x^{\prime}, v^{\prime}\right)\right] \phi(x, v) \\
& +\left[\phi^{\dagger}(x,-v), \phi^{\dagger}\left(x^{\prime},-v^{\prime}\right)\right] \phi\left(x^{\prime}, v^{\prime}\right) \phi(x, v) .
\end{aligned}
$$

Next, using the appropriate field commutators [16] in (4.33), one obtains

$$
\left[O(x, v), O\left(x^{\prime}, v^{\prime}\right)\right]=0 .
$$

Equation (4.34) is consistent with microcausality. However, extremely large relative four-velocities will give rise to significant Planck-scale modifications of the causal domain, (see (4.4) and (4.5)).

5. Conclusions. A theory of a Lorentz-invariant quantum field in the spacetime tangent bundle has been formulated based on maximal-acceleration invariance. The field was demonstrated to have a physically based Planck-scale effective regularization with an effective spectral cutoff near the Planck mass. Also, it was argued that provided the adjoint field is consistently generalized, the necessary commutators are vanishing and the field is microcausal. There are, however, Planck-scale modifications of the causal domain, but they only become significant for extremely large relative four-velocities at the separated space-time points. The causal domain is canonical for vanishing relative fourvelocities. The geometry of the causal domain suggests that at the Planck scale, causal connectivity may occur between spacelike separated points and also at larger scales for extremely large relative four-velocities.

\section{REFERENCES}

[1] M. Abramowitz and I. A. Stegun, Handbook of Mathematical Functions with Formulas, Graphs, and Mathematical Tables, National Bureau of Standards, District of Columbia, 1972.

[2] J. D. Bjorken and S. D. Drell, Relativistic Quantum Fields, McGraw-Hill, New York, 1965.

[3] D. I. Blokhintsev, Space and Time in the Microworld, D. Reidel Publishing, Dordrecht, 1973.

[4] N. N. Bogolubov, A. A. Logunov, A. I. Oksak, and I. T. Todorov, General Principles of Quantum Field Theory, Mathematical Physics and Applied Mathematics, vol. 10, Kluwer Academic Publishers, Dordrecht, 1990.

[5] N. N. Bogolubov, A. A. Logunov, and I. T. Todorov, Introduction to Axiomatic Quantum Field Theory, W. A. Benjamin, Massachusetts, 1975.

[6] H. E. Brandt, Maximal proper acceleration relative to the vacuum, Lett. Nuovo Cimento 38 (1983), 522-524, Erratum in 31 (1984), 192.

[7] _ The maximal acceleration group, 13th International Colloquium on Group Theoretical Methods in Physics (College Park, Md, 1984), World Scientific Publishing, Singapore, 1984, pp. 519-522. 
[8] _ Maximal-acceleration invariant phase space, The Physics of Phase Space (Y. S. Kim and W. W. Zachary, eds.), Springer-Verlag, Berlin, 1987, pp. 414416.

[9] __ Maximal proper acceleration and the structure of spacetime, Found. Phys. Lett. 2 (1989), no. 2, 39-58, Erratum, no. 4, 405.

[10] _ Structure of spacetime tangent bundle, Found. Phys. Lett. 4 (1991), no. 6, 523-536.

[11]__ Finslerian spacetime, Finsler Geometry (Seattle, Wash, 1995), Contemp. Math., vol. 196, American Mathematical Society, Rhode Island, 1996, pp. 273-287.

[12]__ Quantum fields in the spacetime tangent bundle, Found. Phys. Lett. 11 (1998), no. 3, 265-275.

[13] _ Finslerian fields in the spacetime tangent bundle, Chaos Solitons Fractals 10 (1999), no. 2-3, 267-282.

[14] Causal domain of Minkowski-spacetime tangent bundle, Found. Phys. Lett. 13 (2000), no. 6, 581-588.

[15] _ Finslerian fields, Finslerian Geometries (Edmonton, AB, 1998) (P. L. Antonelli, ed.), Fund. Theories Phys., vol. 109, Kluwer Academic Publishers, Dordrecht, 2000, pp. 131-138.

[16] _ Finslerian quantum fields and microcausality, Found. Phys. Lett. 13 (2000), no. 4, 307-328.

[17]___ Particle geodesics and spectra in the spacetime tangent bundle, Rep. Math. Phys. 45 (2000), no. 3, 389-405.

[18] L. S. Brown, Quantum Field Theory, Cambridge University Press, Cambridge, 1992.

[19] A. Erdélyi, W. Magnus, F. Oberhettinger, and F. G. Tricomi, Tables of Integral Transforms. Vol. I, McGraw-Hill, New York, 1954.

[20] R. P. Feynman, A relativistic cut-off for classical electrodynamics, Phys. Rev.(2) 74 (1948), 939-946.

[21] _ The theory of positrons, Phys. Rev. 76 (1949), 749-759.

[22] W. Greiner and J. Reinhardt, Field Quantization, Springer-Verlag, Berlin, 1996.

[23] K. Huang, Quantum Field Theory. From Operators to Path Integrals, A WileyInterscience Publication, John Wiley \& Sons, New York, 1998.

[24] S. S. Schweber, An Introduction to Relativistic Quantum Field Theory, Foreword by Hans A. Bethe, Row, Peterson and Company, Illinois, 1961.

[25] R. F. Streater and A. S. Wightman, PCT, Spin and Statistics, and All That, Advanced Book Classics, Addison-Wesley Publishing, California, 1989.

[26] E. C. G. Stueckelberg, La mécanique du point matériel en théorie de relativité et en théorie des quanta, Helv. Phys. Acta 15 (1942), 23-37 (French).

[27] S. Weinberg, The Quantum Theory of Fields. Vol. I. Foundations, Cambridge University Press, Cambridge, 1995.

[28] E. W. Weisstein, CRC Concise Encyclopedia of Mathematics, CRC Press, Florida, 1999.

Howard E. Brandt: US Army Research Laboratory, 2800 Powder Mill Road, Adelphi, MD 20783, USA

E-mail address: hbrandt@ar1.army.mi 1 


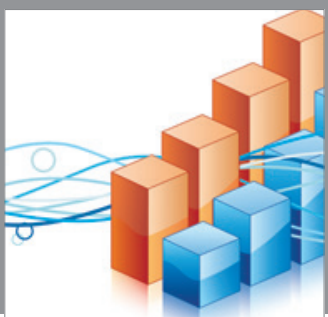

Advances in

Operations Research

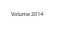

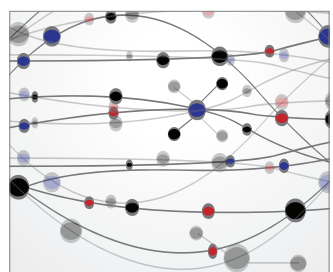

\section{The Scientific} World Journal
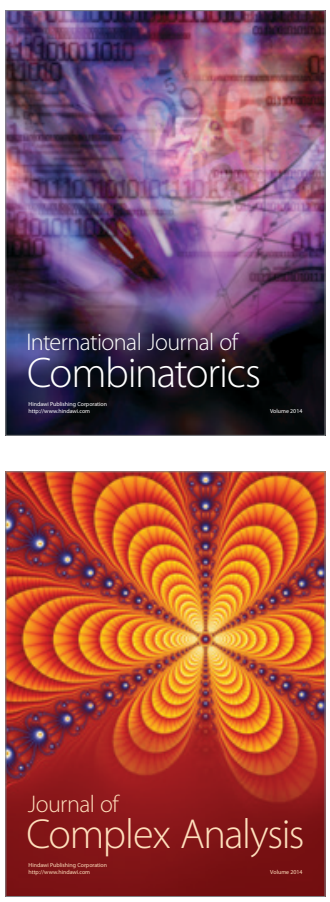

International Journal of

Mathematics and

Mathematical

Sciences
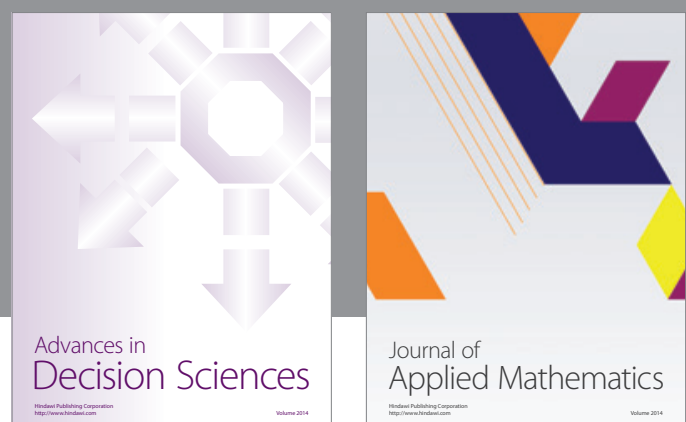

Journal of

Applied Mathematics
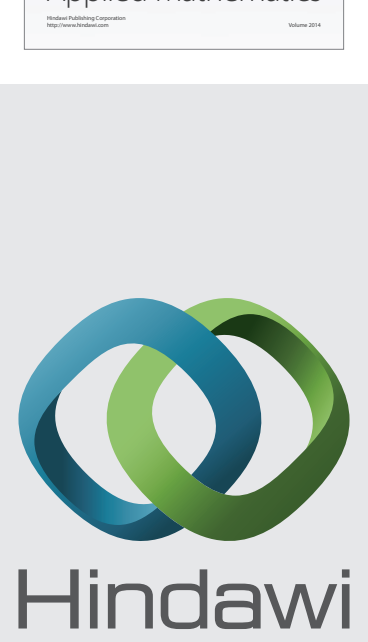

Submit your manuscripts at http://www.hindawi.com
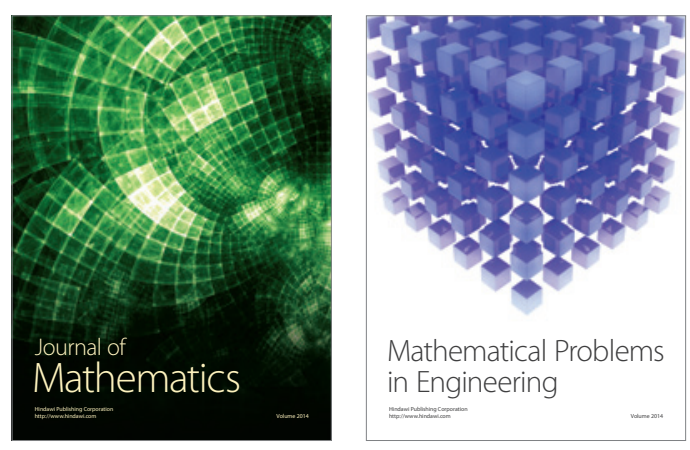

Mathematical Problems in Engineering
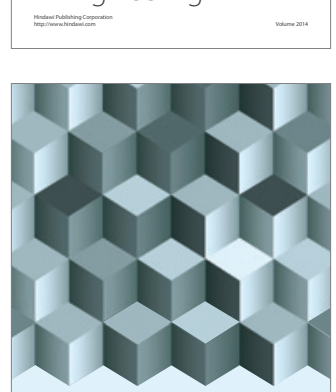

Journal of

Function Spaces
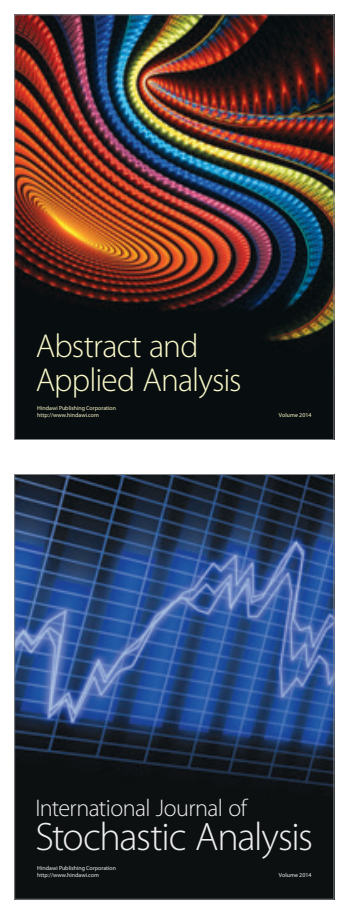

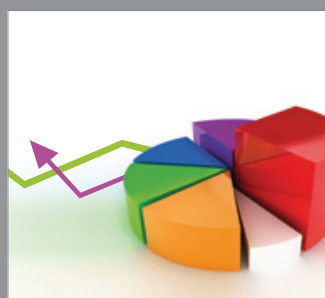

ournal of

Probability and Statistics

Promensencen
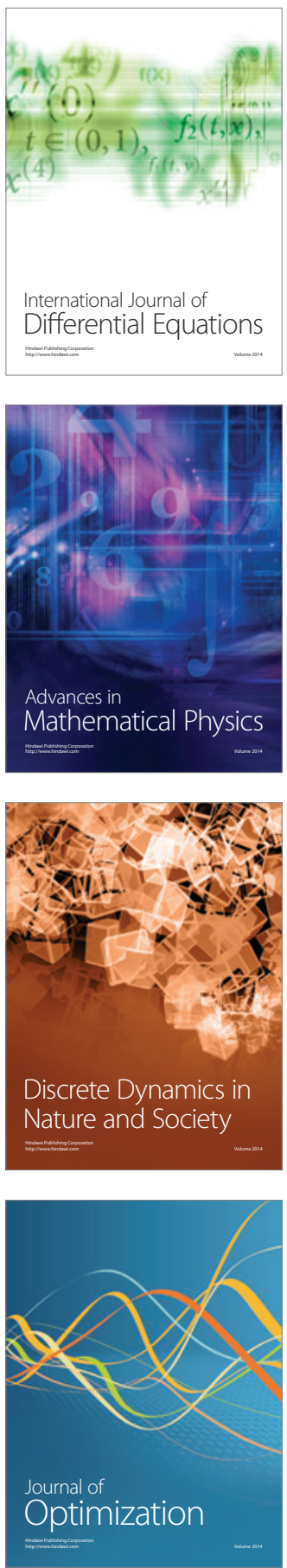